\title{
Risk factors of esophageal fistula induced by re-radiotherapy for recurrent esophageal cancer with local primary site
}

\author{
Xinran Wang ${ }^{1 \dagger}$, Bing Hu${ }^{2 \dagger}$, Jinhu Chen ${ }^{1}$, Feihong Xie ${ }^{1}$, Dan Han ${ }^{1}$, Qian Zhao ${ }^{1}$, Hongfu Sun ${ }^{1}$, Chengrui Fu', \\ Chengxin Liu' ${ }^{1}$, Zhongtang Wang ${ }^{1}$, Haiqun Lin ${ }^{1}$ and Wei Huang ${ }^{1 *}$
}

\begin{abstract}
Purpose: The purpose of the present study was to investigate risk factors for esophageal fistula (EF) in patients with recurrent esophageal cancer receiving re-radiotherapy with or without chemotherapy.

Methods: We reviewed retrospectively the clinical characters and dosimetric parameters of 96 patients with recurrent esophageal cancer treated with re-radiotherapy in Cancer Hospital Affiliated to Shandong First Medical University between August 2014 and January 2021. Univariate and multivariate logistic regression analyses were provided to determine the risk factors of EF induced by re-radiotherapy.

Results: The median time interval between two radiotherapy was 23.35 months (range, 4.30 to 238.10 months). EF occurred in 19 patients (19.79\%). In univariate analysis, age, T stage, the biologically equivalent dose in the reradiotherapy, total biologically equivalent dose, hyperfractionated radiotherapy, ulcerative esophageal cancer, the length of tumor and the maximum thickness of tumor had a correlation with the prevalence of EF. In addition, age $(H R=0.170,95 \% \mathrm{Cl} 0.030-0.951, p=0.044)$, T stage $(H R=8.369,95 \% \mathrm{Cl} 1.729-40.522, p=0.008)$, ulcerative esophageal cancer ( $\mathrm{HR}=5.810,95 \% \mathrm{Cl} 1.316-25.650, p=0.020)$ and the maximum thickness of tumor $(\mathrm{HR}=1.314,95 \% \mathrm{Cl} 1.098-$ $1.572, p=0.003)$ were risk factors of EF in multivariate logistic regression analysis.

Conclusions: The incidence of EF was significantly increased in patients with recurrent esophageal cancer who underwent re-radiotherapy. This study revealed that age, T stage, ulcerative esophageal cancer and the maximum thickness of the tumor were risk factors associated with EF. In clinical work, patients with risk factors for EF ought to be highly concerned and individualized treatment plans should be taken to reduce the occurrence of EF.
\end{abstract}

Keywords: Esophageal cancer, Esophageal fistula, Radiotherapy, Risk factor

\section{Background}

Loco-regional recurrence is the main type of failure in patients with esophageal cancer (EC) following chemoradiotherapy (CRT). Loco-regional recurrence is very

\footnotetext{
*Correspondence: alvinbird@126.com

${ }^{+}$Xinran Wang and Bing Hu are joint first authors.

1 Shandong Cancer Hospital and Institute, Shandong First Medical University and Shandong Academy of Medical Sciences, No.440, Jiyan road, Huaiyin distract, Jinan 250117, Shandong Province, China

Full list of author information is available at the end of the article
}

common, occurring in approximately $40-60 \%$ of patients $[1,2]$. Once recurrence occurs, most patients lost the chance of surgery [3, 4]. The prognosis of recurrent patients is poor and the mortality is high. Patients will die without treatment within 1 year [5]. The 5-year survival rate is only $0-11 \%[6,7]$.

It is difficult to treat those patients with recurrent esophageal cancer (REC) after primary radiotherapy (RT). There are no general treatment guidelines for REC after primary RT. In patients with advanced REC, 
the effects of tumor recurrence are extremely distressing, and the main purpose of treatment is to relieve the patients' dysphagia. Chemotherapy is a palliative treatment, which rarely achieves remission of the lesion. Re-radiotherapy (re-RT) appear to be an important treatment for local recurrence of EC after primary RT. The use of re-RT can significantly alleviate the symptoms of dysphagia, thereby improving the survival time and quality of life of patients [8].

The high incidence of complications of re-RT is a major problem especially esophageal fistula (EF), which is one of the serious complications. Anatomically, the esophagus is a muscular tube without serosa layer. Therefore, local extension of tumor to adjacent structure is common due to the lack of barrier to loco-regional spread such as the pericardium, trachea, mediastinum [9]. In addition, CRT can induce EF because of the imbalance between tumor shrinkage and normal tissue repair $[10,11]$. EF can easily lead to serious infections, including pneumonia, lung abscess and sepsis. The mortality of patients with EF is high. Most patients with EF die within 3-4months $[12,13]$. Therefore, early prevention, early diagnosis and early treatment of EF are very important. The incidence of EF in EC patients receiving CRT has been reported to be $6-22 \%$ [14]. However, there are few reports on risk factors of EF caused by re-RT for REC patients. We conducted this study to answer this question.

\section{Materials and methods}

\section{Patients' selection}

This study retrospectively analyzed 96 patients who were treated with re-RT in Cancer Hospital Affiliated to Shandong First Medical University between August 2014 and January 2021. The eligibility criteria were as follows: 1 . All patients with pathologically confirmed REC with local primary site;2. Re-staged as II-IV based on the American Joint Committee on Cancer (7th edition);3. Karnofsky performance status (KPS) score $\geq 70 ; 4$. Treated by primary RT or re-RT with or without chemotherapy;5. The target area of primary RT and re-RT partially overlapped;6. Patients without any other serious medical illness except EC.7. No EF before re-RT. The exclusion criteria were as follows: 1. Patients underwent esophageal surgery previously; 2 . Lost to follow-up. It should be noted that this study only included tumor recurrence in the primary tumor bed, with or without lymph nodes recurrence.

\section{Pretreatment evaluation}

All patients underwent a physical examination, barium esophagography, fiber esophagoscopy, endoscopic ultrasonography, pathological and cytological examination, the cervical, chest and abdomen contrast-enhanced computed tomography (CT), magnetic resonance imaging (MRI) of the head. The diagnosis of recurrence after the primary RT for EC was based on pathological examination. The $\mathrm{T}$ stage was diagnosed by oncologists and radiologists based on findings of contrast-enhanced CT and endoscopic ultrasonography. The maximum thickness of the tumor was measured with MRI, CT or/and Positron Emission Tomography-Computer Tomography (PET-CT) by taking the maximum thickness of internal diameter and external diameter. The tumor length was determined by barium esophagography, esophagoscope, CT, MRI, or/and PET-CT. Esophageal stenosis is based on the patient's clinical symptoms combined with the measurement results of barium esophagography or esophagoscopy. The time interval between two RTs was defined as from the end of primary RT to the beginning of re-RT.

\section{Treatment programs}

All patients with REC included in the study were treated with concurrent CRT, sequential CRT or RT alone.

\section{Radiotherapy}

All patients underwent re-RT. Each patient was placed in supine position with a body vacuum bag or head and neck thermoplastics technology, raising both arms and crossing elbows. The scanning range was from the ring membrane to $5 \mathrm{~cm}$ below the lower edge of the lungs, a slice thickness of $3.0 \mathrm{~mm}$. The CT image was transmitted to the Varian planning system, radiologists and radiation oncologists collectively delineate the target area and the endangered organ. The gross tumor volume (GTV) included recurrent tumor lesions and metastatic lymph nodes that could be seen on CT/PET-CT/MRI. The clinical target volume (CTV) was subclinical lesions and high-risk lymphatic drainage areas [15]. The planning target volume (PTV) was defined as $0.5-0.8 \mathrm{~cm}$ beyond the CTV. Radiation was administered via a 6 MV X-ray, and 3 to 6 irradiation fields IMRT were used to pass the dose. The volume histogram was optimized, 95\% isodose line covered the planned target area, 73 patients $(76.04 \%)$ received conventional fractionated RT with the median dose of $50.4 \mathrm{~Gy}$ (16.0-61.2Gy), 1.8-2.0 Gy / time, 5 times / week; 23 patients (23.96\%) received hyperfractionated $\mathrm{RT}$ with the median dose of 50.4 Gy (31.2-60.0 Gy), 1.15-1.30 Gy / time, twice a day. Regarding the lungs, the V20 and mean dose were limited within $30 \%$ and 20 Gy respectively in the first treatment, after recurrence V20 was less than $25 \%$. The highest dose of the spinal cord was $<25 \mathrm{~Gy}$, and the mean dose of the heart was $\leq 30 \mathrm{~Gy}$. 


\section{Chemotherapy}

Patients with REC generally chose the following two chemotherapy regimens: PF scheme include 5 -fluorouracil $(5-\mathrm{FU}) 1000 \mathrm{mg} / \mathrm{m}^{2}$ on days $1-5$ or $\mathrm{S}-160-80 \mathrm{mg} / \mathrm{m}^{2}$ on days $1-14$ plus cisplatin (DDP) $25 \mathrm{mg} / \mathrm{m}^{2}$ on days $1-3$. DP scheme include docetaxel (TXT) $75 \mathrm{mg} / \mathrm{m}^{2}$ or paclitaxel 135-150 mg on day one combined with DDP $25 \mathrm{mg} /$ $\mathrm{m}^{2}$ on days $1-3$. Both schemes were repeated every 21-28 days.

\section{Diagnostic criteria of EF}

Common symptoms of EF include severe cough caused by consuming water or food, chest pain and fever. Discovery of fistulas by barium esophagography or/and esophageal endoscopy is the gold standard for the diagnosis of EF. Barium esophagography shows that the contrast medium entered the trachea, mediastinum or aorta through the fistula (see Fig. 1). CT is also an important method for the diagnosis of EF (see Fig. 2). Types of EF include esophageal-mediastinum fistula (EMF), esophago-respiratory fistula (ERF) and aorto-esophageal fistula (AEF). In this study, no patients developed AEF.

\section{Data collection}

The following clinical characters and dosimetric parameters were collected and analyzed. Clinical characters include age, gender, location of the tumor and stage, the length of tumor, the maximum thickness of the tumor, esophageal stenosis, the time interval between two RTs,

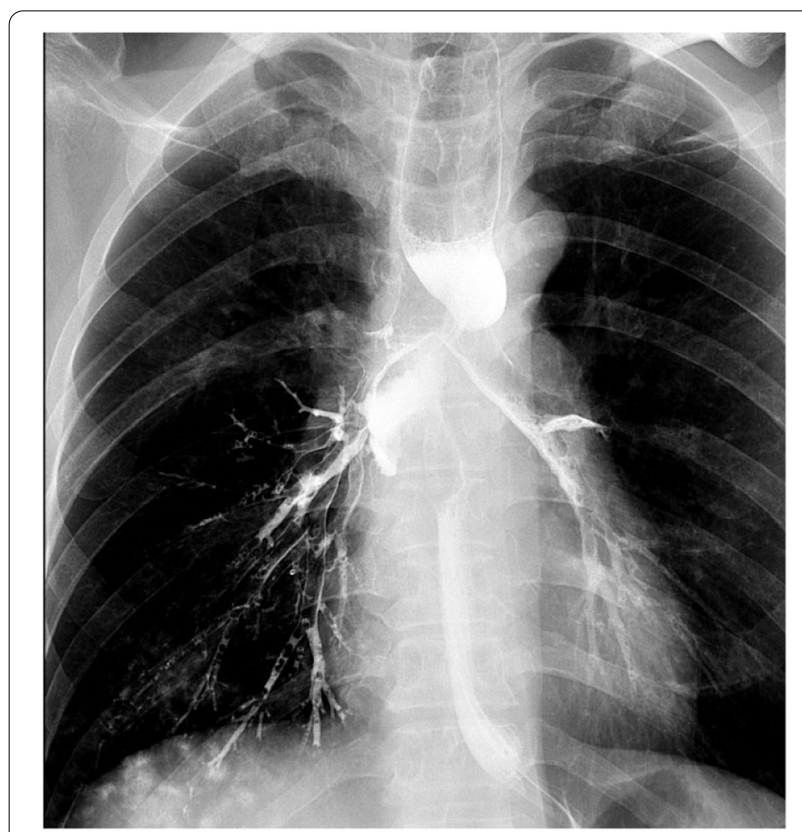

Fig. 1 Esophagus barium meal examination shows esophago-respiratory fistula

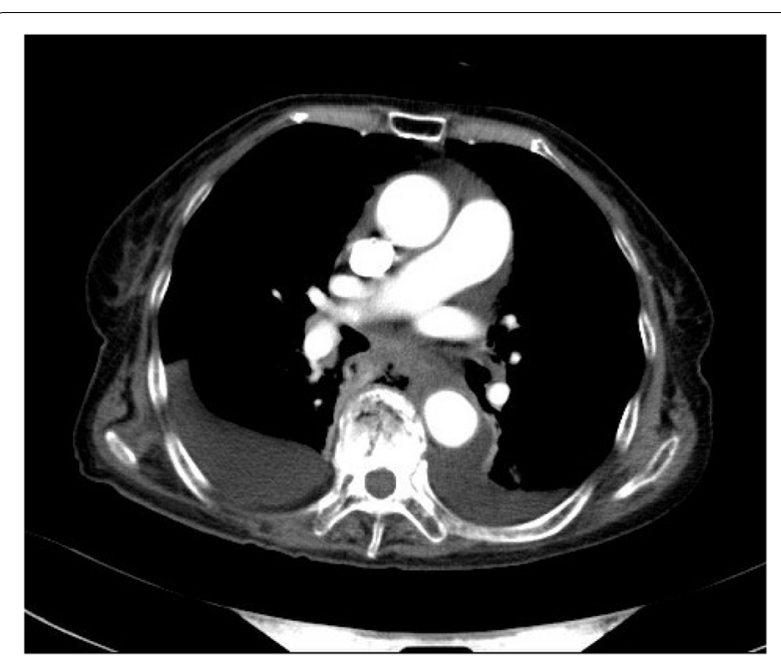

Fig. 2 CT scan of the chest shows esophageal-mediastinum fistula

ulcerative EC, concurrent CRT in primary RT, concurrent CRT in re-RT. Dosimetric parameters include the biologically equivalent dose (BED) in re-RT, the total BED and hyperfractionated RT in re-RT.

\section{Statistical analysis}

Retrospectively summarized and analyzed datum from all patients. The incidence of EF was calculated for all patients during or after RT. Univariate analysis was performed for 15 variables by logistic regression methods. Next, to select informative risk factors, the meaningful variables $(P$-value $<0.1)$ detected by univariate analysis were subjected to multivariate analysis. Univariate and multivariate analyses were carried out using logistic regression to estimate the odds ratio (OR) and 95\% confidence intervals (CIs). $P$-value $<0.05$ was considered statistically significant. All analyses were performed using IBM SPSS Statistics version 23.

\section{Follow-up}

The last follow-up was in May 2021, and the median follow-up period was 14.80 months (range 0.3390.83 months). The follow-up rate was $100 \%$ based on medical records, outpatient records, and telephone follow-up. Follow-up assessments were performed every 3 months in the first 2 year, followed every 6 months. At each follow-up visit, evaluation including physical examination, contrast-enhanced CT of the cervical region, chest, and abdomen and barium esophagography.

\section{Results}

\section{Patient features}

In this study, 96 patients were enrolled. EF was observed in 19 patients, and the incidence of EF was $19.79 \% .3$ 
patients developed EF during re-RT and 16 patients experienced $\mathrm{EF}$ after re-RT. The median time interval between the date of re-RT completion and EF diagnosis was 3.2 months (range, 0.6 to 9.3 months). The specific characteristics of patients were listed in Table 1.

\section{Survival}

The Kaplan-Meier method was used to calculate the survival time from the first day of diagnosis of recurrence to the day of death, seen Fig. 3. Overall survival considered deaths from any cause. The median survival

Table 1 General clinical information of patients

\begin{tabular}{|c|c|c|}
\hline Characteristics & Number of patients $(N=96)$ & Number of EF patients $(N=19)$ \\
\hline \multicolumn{3}{|l|}{ Age (years) } \\
\hline$<70$ & $59(61.46 \%)$ & $16(84.21 \%)$ \\
\hline$\geqq 70$ & $37(38.54 \%)$ & $3(15.79 \%)$ \\
\hline \multicolumn{3}{|l|}{ Gender } \\
\hline Female & $23(23.96 \%)$ & $2(10.53 \%)$ \\
\hline Male & $73(76.04 \%)$ & $17(89.47 \%)$ \\
\hline \multicolumn{3}{|l|}{ Tstage } \\
\hline Non-T4 & $65(67.71 \%)$ & $8(42.11 \%)$ \\
\hline T4 & $31(32.29 \%)$ & $11(57.89 \%)$ \\
\hline \multicolumn{3}{|l|}{ TNM clinical stage } \\
\hline$\|A-\| B$ & $30(31.25 \%)$ & $5(26.32 \%)$ \\
\hline$\|A-\| C$ & $43(44.79 \%)$ & $6(31.58 \%)$ \\
\hline IV & $23(23.96 \%)$ & $8(42.1 \%)$ \\
\hline \multicolumn{3}{|l|}{ Location of tumor } \\
\hline Cervical section & $10(10.42 \%)$ & $1(5.26 \%)$ \\
\hline Upper thoracic & $38(39.58 \%)$ & $12(63.16 \%)$ \\
\hline Mid thoracic & $32(33.33 \%)$ & $3(15.79 \%)$ \\
\hline Lower thoracic & $16(16.67 \%)$ & $3(15.79 \%)$ \\
\hline Concurrent $\mathrm{CRT}$ in primary $\mathrm{RT}$ & $30(31.25 \%)$ & $5(26.32 \%)$ \\
\hline Concurrent CRT in re-RT & $26(27.08 \%)$ & $5(26.32 \%)$ \\
\hline Median BED in re-RT & $59.47(19.20-74.34)$ & $54.00(31.20-61.20)$ \\
\hline Median total BED & $131.47(84.00-155.15)$ & $135.72(104.60-153.60)$ \\
\hline \multicolumn{3}{|l|}{ Hyperfractionated RT in re-RT } \\
\hline No & $73(76.04 \%)$ & $18(94.74 \%)$ \\
\hline Yes & $23(23.96 \%)$ & $1(5.26 \%)$ \\
\hline \multicolumn{3}{|l|}{ Ulcerative EC } \\
\hline No & $69(71.88 \%)$ & $10(52.63 \%)$ \\
\hline Yes & $27(28.12 \%)$ & $9(47.37 \%)$ \\
\hline \multicolumn{3}{|l|}{ Esophageal stenosis (cm) } \\
\hline$<0.5$ & $23(23.96 \%)$ & $3(15.79 \%)$ \\
\hline $0.5-1$ & $67(69.79 \%)$ & $15(78.95 \%)$ \\
\hline$\geq 1$ & $6(6.25 \%)$ & $1(5.26 \%)$ \\
\hline Median the length of tumor $(\mathrm{cm})$ & 4.35 & 5 \\
\hline The length of tumor (range) $(\mathrm{cm})$ & $(2-11)$ & $(3-10)$ \\
\hline Median the maximum thickness of tumor (mm) & 14.685 & 17.24 \\
\hline The maximum thickness of tumor (range) (mm) & $(7.17-29.61)$ & $(12.75-29.61)$ \\
\hline Median the time interval between two RTs (months) & 23.35 & 24.4 \\
\hline The time interval between two RTs (range) (months) & $(4.30-238.10)$ & $(8.87-61.27)$ \\
\hline \multicolumn{3}{|l|}{ Type of EF } \\
\hline EMF & & $8(42.11 \%)$ \\
\hline ERF & & $11(57.89 \%)$ \\
\hline AEF & & 0 \\
\hline
\end{tabular}

EF Esophageal fistula, CRT Chemoradiotherapy, RT Radiotherapy; re-RT re-radiotherapy, BED Biologically equivalent dose, EC Esophageal cancer, EMF Esophagealmediastinum fistula, ERF Esophago-respiratory fistula, $A E F$ Aorto-esophageal fistula 


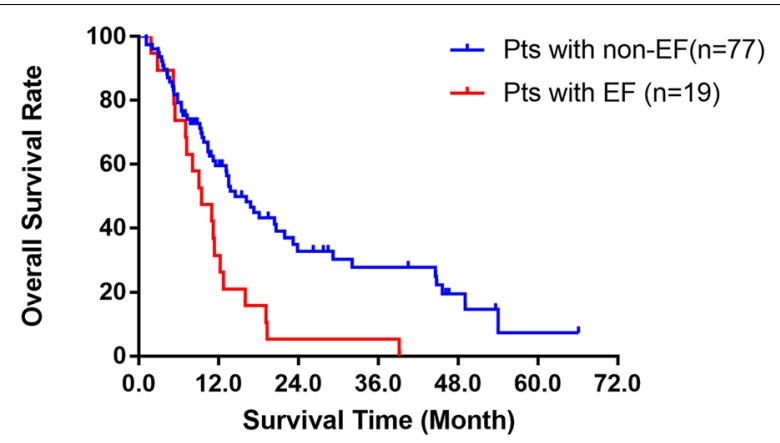

Fig. 3 There was a significant difference between overall survival rates in patients with non-EF and in patients with EF (Kaplan-Meier method)

time (MST) of 77 patients with non-EF was 14.5 months (95\% CI: 10.302-18.698), and the 6-month, 1-year and 2-year overall survival rates were 79.2,59.6 and 32.9\%, respectively. The 6-month 1-year and 2-year overall survival rates in the 19 patients with EF were 73.7,31.6 and $5.3 \%$, respectively, with an MST of 9.4 months (95\% CI: 5.371-13.429). There was a significant difference between survival rates in the two groups (log-rank test, $p=0.0016)$. In the previous study of EC patients who underwent RT with or without chemotherapy [16], the MST of patients without EF and patients with EF were 36.8 vs 5.3 months, respectively. The prognosis of patients with EF was very poor, and all EF patients died during the follow-up period.

\section{Risk factors for EF}

In the univariate analysis, age, $\mathrm{T}$ stage, the BED in re-RT, total BED, hyperfractionated RT in re-RT, ulcerative $\mathrm{EC}$, the length of tumor and the maximum thickness of tumor were selected as meaningful factors for $E F$. The results of univariate analysis of risk factors for EF were shown in Table 2. The meaningful factors were included in multivariate analysis. Age, T stage, ulcerative EC and the maximum thickness of tumor had a significant correlation with the incidence of EF. The detailed information was shown in Table 3.

\section{Discussion and conclusions}

The local recurrence after primary RT in patients with $\mathrm{EC}$ is a tough challenge for clinical oncologists, it was as high as $66.5 \%$ after RT with or without chemotherapy in 2 years [17]. The vast majority of patients with REC have missed the opportunity for radical surgery, re-RT may be an effective modality [18]. The condition of some patients could be under long-term control, and the overall survival rate and survival rate after relapse could be improved. But EF is one of the serious complications, which is the main cause of treatment failure and death. The incidence for this event was reported to be $18-20 \%[3,19]$. In the same center, $\mathrm{Xu}$ et al. [20] reported that ECOG PS, BMI, T4, N2/3 and re-RT were independent factors for EF, then a nomogram was constructed and externally validated for the prediction of EF associated with RT. In our previous study [16], we also analyzed the risk factors associated with EF after RT for esophageal squamous cell carcinoma, it was found that T4 stage, N3 stage, re-RT, ulcerative EC, esophageal stricture and maximum tumor thickness were risk factors for EF. Among these factors re-RT was a strong risk factor for EF. Thus, we conducted this research to confirm the risk factors for EF in patients with REC receiving re-RT. In total, 15 clinical and dosimetric factors were included in the analysis. Age, T stage, ulcerative $\mathrm{EC}$ and the maximum thickness of tumor were revealed as risk factors for fistula formation.

Han et al. [21] reported that of 20 patients with EF, 14 of them were caused by RT. Esophageal perforation caused by RT is mainly due to the imbalance between the regression speed of tumor tissue and the repair speed of normal tissue. The rapid regression of tumor is related to the sensitivity of tumor for radiation, dose and speed of radiation. Kim et al. [3] reported that 17 patients with REC received re-RT, and 3 patients developed EF (17.65\%). Zhou et al. [19] also reported on the efficacy and feasibility of salvage RT in patients with locally REC after radical CRT, this study showed that although re-RT could prolong the survival time of patients, the incidence of EF was as high as $20 \%(11 / 55)$. In our study, there were 19 patients with EF. The probability of EF in patients receiving re-RT was higher than that in patients receiving primary RT.

Esophagus tumor has a strong invasion to surrounding tissues and adjacent organs, which is related to the high incidence of EF [22]. Especially in T4 stage, the tissues and organs around the esophagus are more severely invaded. However, the esophagus surrounds the aorta, trachea, bronchus and mediastinum. The tumor can not only invade the esophageal wall, but invade the surrounding tissues and organs to form EF as well. The EF rates reported in T4 patients receiving CRT was in the range of $10-12 \%[14,23]$. In this study, the incidence of EF in $\mathrm{T} 4$ patients receiving re-RT was $57.89 \%$, which greatly increased the risk of EF. Therefore, more attention should be paid to patients with T4 stage. Our results also found that the larger maximum thickness of the tumor was prone to EF. We analyzed that it might be related to the fact that the thickness of the tumor determined the irradiation area, which in turn affected the irradiation dose of important organs around the esophagus, leading to this serious complication. But the BED in re-RT and the total BED were not statistically significant in the occurrence of 
Table 2 Results of univariate analysis of risk factors for EF

\begin{tabular}{|c|c|c|c|c|c|}
\hline Characteristics & EF- & $\mathrm{EF}+$ & OR & $95 \% \mathrm{Cl}$ & $P$-value \\
\hline \multicolumn{6}{|l|}{ Age (years) } \\
\hline$<70$ & 43 & 16 & 1 & & \\
\hline$\geqq 70$ & 34 & 3 & 0.237 & $0.064-0.881$ & 0.032 \\
\hline \multicolumn{6}{|l|}{ Gender } \\
\hline Female & 21 & 2 & 1 & & \\
\hline Male & 56 & 17 & 3.187 & $0.677-14.997$ & 0.142 \\
\hline \multicolumn{6}{|l|}{ Tstage } \\
\hline Non-T4 & 57 & 8 & 1 & & \\
\hline T4 & 20 & 11 & 3.919 & $1.380-11.126$ & 0.010 \\
\hline \multicolumn{6}{|l|}{ TNM clinical stage } \\
\hline$\|A-\| B$ & 25 & 5 & 1 & & \\
\hline$\|I A-\| \mid C$ & 37 & 6 & 0.811 & $0.223-2.948$ & 0.750 \\
\hline IV & 15 & 8 & 2.667 & $0.736-9.665$ & 0.135 \\
\hline \multicolumn{6}{|l|}{ Location of tumor } \\
\hline Cervical section & 9 & 1 & 1 & & \\
\hline Upper thoracic & 26 & 12 & 4.154 & $0.471-36.609$ & 0.200 \\
\hline Mid thoracic & 29 & 3 & 0.931 & $0.086-10.095$ & 0.953 \\
\hline Lower thoracic & 13 & 3 & 2.077 & $0.185-23.298$ & 0.553 \\
\hline Concurrent CRT in primary RT & 25 & 5 & 0.743 & $0.241-2.293$ & 0.605 \\
\hline Concurrent CRT in re-RT & 21 & 5 & 0.952 & $0.305-2.971$ & 0.933 \\
\hline Median the BED in re-RT & 59.47 & 54 & & & \\
\hline The BED in re-RT (range) & $(19.20-72.0)$ & $(31.20-61.20)$ & 1.056 & $1.991-1.126$ & 0.094 \\
\hline Median the total BED & 131.47 & 135.72 & & & \\
\hline The total BED (range) & $(84.0-155.15)$ & $(104.60-153.60)$ & 1.046 & $1.996-1.099$ & 0.072 \\
\hline \multicolumn{6}{|l|}{ Hyperfractionated RT in re-RT } \\
\hline No & 55 & 18 & 1 & & \\
\hline Yes & 22 & 1 & 0.139 & $0.017-1.105$ & 0.062 \\
\hline \multicolumn{6}{|l|}{ Ulcerative EC } \\
\hline No & 59 & 10 & 1 & & \\
\hline Yes & 18 & 9 & 2.95 & $1.039-8.378$ & 0.042 \\
\hline \multicolumn{6}{|l|}{ Esophageal stenosis (cm) } \\
\hline$<0.5$ & 20 & 3 & 1 & & \\
\hline $0.5-1$ & 52 & 15 & 1.923 & $0.502-7.363$ & 0.340 \\
\hline$\geq 1$ & 5 & 1 & 1.333 & $0.113-15.704$ & 0.819 \\
\hline Median the length of tumor $(\mathrm{cm})$ & 4 & 5 & & & \\
\hline The length of tumor (range) $(\mathrm{cm})$ & $(2-11)$ & $(3-10)$ & 1.314 & $1.039-1.663$ & 0.023 \\
\hline Median the maximum thickness of tumor (mm) & 13.54 & 17.24 & & & \\
\hline The maximum thickness of tumor (range) (mm) & $(7.17-24.87)$ & $(12.75-29.61)$ & 1.226 & $1.084-1.387$ & 0.001 \\
\hline Median the time interval between two RTs (months) & 23.33 & 24.4 & & & \\
\hline The time interval between two RTs (range) (months) & $(4.30-238.10)$ & $(8.87-61.27)$ & 0.989 & $0.974-1.003$ & 0.131 \\
\hline
\end{tabular}

EF Esophageal fistula, CRT Chemoradiotherapy, $R T$ Radiotherapy, re- $R T$ re-radiotherapy, $B E D$ Biologically equivalent dose, EC Esophageal cancer, $C I$ Confidence interval, $O R$ Odds ratio

EF. For patients with REC, the suitable irradiation dose of remains uncertain, and further research is needed. We recommend that the total dose be as low as possible as higher dose was reported to increase the risk of perforation [24]. Our study revealed that the incidence of EF was relatively higher and statistically significant in patients with ulcerative EC than those with non-ulcerative EC. In the study of Tsushima et al. [25] 100\% of patients with EF had ulcerative tumor. It was suggested that ulcerative EC was more prone to EF. Statistical analysis also showed that age $<70$ was a risk factor for EF. Compared with conventional RT, this study found that 23 patients underwent 
Table 3 Results of multivariate analysis of risk factors for EF

\begin{tabular}{|c|c|c|c|}
\hline Characteristics & OR & $95 \% \mathrm{Cl}$ & $P$-value \\
\hline \multicolumn{4}{|l|}{ Age (years) } \\
\hline$<70$ & 1 & & \\
\hline$\geqq 70$ & 0.170 & $0.030-0.951$ & 0.044 \\
\hline \multicolumn{4}{|l|}{ T stage } \\
\hline Non-T4 & 1 & & \\
\hline T4 & 8.369 & $1.729-40.522$ & 0.008 \\
\hline The BED in re-RT & 1.063 & $0.918-1.231$ & 0.416 \\
\hline The total BED & 0.986 & $0.881-1.102$ & 0.798 \\
\hline \multicolumn{4}{|l|}{ Hyperfractionated RT in re-RT } \\
\hline No & 1 & & \\
\hline Yes & 0.091 & $0.006-1.417$ & 0.087 \\
\hline \multicolumn{4}{|l|}{ Ulcerative EC } \\
\hline No & 1 & & \\
\hline Yes & 5.810 & $1.316-25.650$ & 0.020 \\
\hline The length of tumor (cm) & 1.138 & $0.818-1.585$ & 0.443 \\
\hline $\begin{array}{l}\text { The maximum thickness of } \\
\text { tumor }(\mathrm{mm})\end{array}$ & 1.314 & $1.098-1.572$ & 0.003 \\
\hline
\end{tabular}

$R T$ Radiotherapy, re-RT re-radiotherapy, $B E D$ Biologically equivalent dose, $E C$ Esophageal cancer, $\mathrm{Cl}$ Confidence interval, $O R$ Odds ratio

re-RT using hyperfractionated treatment modality, of which only 1 patient developed EF. However, whether hyperfractionation modality can reduce the incidence of EF needs to be verified in future randomized clinical surveys.

There were several limitations in this retrospective study including a smaller number of cases and the shorter follow-up period. Second, it was difficult to accurately distinguish between treatment-related EF and EF resulting from tumor progression, and finally, this was a study from a single center.

In conclusion, this study showed that age, $\mathrm{T}$ stage, ulcerative EC and the maximum thickness of the tumor were closely related to EF. Once EF occurs the prognosis is highly poor, no matter what kind of treatment strategy the effect is not good. Thus, the focus is on prevention. We should carefully formulate individualized treatment plans, highly select patients suitable for re-RT, strengthen adjuvant treatment, and minimize the risk of EF. In recent years, tumor immunotherapy has become a research hotspot of scholars at home and abroad. In the next study, we can explore whether immunotherapy combined with RT will increase the risk of EF.

\section{Abbreviations}

EC: Esophageal cancer; CRT: Chemoradiotherapy; REC: Recurrent esophageal cancer; RT: Radiotherapy; re-RT: Re-radiotherapy; EF: Esophageal fistula; KPS: Karnofsky performance status; CT: Computed tomography; MRI: Magnetic resonance imaging; PET-CT: Positron Emission Tomography-Computer Tomography; GTV: Gross tumor volume; CTV: Clinical target volume; PTV: planning target volume; TXT: Docetaxel; DDP: Cisplatin; 5-FU: 5-fluorouracil; EMF: Esophageal-mediastinum fistula; ERF: Esophago-respiratory fistula; AEF: Aorto-esophageal fistula; BED: Biologically equivalent dose; OR: Odds ratio; Cls: Confidence intervals; MST: Median survival time.

\section{Acknowledgements}

Not applicable.

\section{Authors' contributions}

$\mathrm{XW}$ and $\mathrm{BH}$ were responsible for research design, planning implementation, statistical analysis, and drafted the manuscript. JC and FX collected important background information and carried out the data acquisition. DH and HS participated in study design and data acquisition. QZ and CL carried out literature search and data aggregation. ZW and HL provided the theoretical proof and academic advice. WH is responsible for the topic selection, overall research guidance, and revision of the paper. All authors contributed to the article and approved the submitted version.

\section{Funding}

This work was supported by grants from National Natural Science Foundation of China [81773232]; Academic Promotion Program of Shandong First Medical University (Shandong Academy of Medical Sciences)[2020RC002] and Project of Young Taishan Scholars [Tsqn201909187].

\section{Availability of data and materials}

The datasets used and analyzed during the current study are available from the corresponding author on reasonable request.

\section{Declarations}

Consent to publication

Not applicable.

\section{Ethics approval and consent to participate}

All procedures of the study were in accordance with the 1964 Declaration of Helsinki and its later amendments or with comparable ethical standards. The study was approved by the Ethics Committee of Cancer Hospital Affiliated to Shandong First Medical University (no SDTHEC2022001008). For this retrospective study, the need of the informed consent was exempted by the Ethics Committee of Cancer Hospital Affiliated to Shandong First Medical University, and all data were kept confidential.

\section{Competing interests}

There is no conflict of interest in this manuscript.

\section{Author details}

'Shandong Cancer Hospital and Institute, Shandong First Medical University and Shandong Academy of Medical Sciences, No.440, Jiyan road, Huaiyin distract, Jinan 250117, Shandong Province, China. ${ }^{2}$ Department of Oncology, Jinxiang people's hospital, Jinxiang, Shandong Province, China.

Received: 22 August 2021 Accepted: 21 February 2022

Published online: 24 February 2022

\section{References}

1. Cooper JS, Guo MD, Herskovic A, et al. Chemoradiotherapy of locally advanced esophageal cancer: long-term follow-up of a prospective randomized trial (RTOG 85-01). radiation therapy oncology group. JAMA. 1999;281(17):1623-7.

2. Pennathur A, Gibson MK, Jobe BA, Luketich JD. Oesophageal carcinoma. Lancet. 2013;381 (9864):400-12.

3. Kim YS, Lee CG, Kim KH, et al. Re-irradiation of recurrent esophageal cancer after primary definitive radiotherapy. Radiat Oncol J. 2012;30(4):182-8.

4. Yamaguchi $\mathrm{S}$, Ohguri $\mathrm{T}$, Imada $\mathrm{H}$, et al. Multimodal approaches including three-dimensional conformal re-irradiation for recurrent or persistent esophageal cancer: preliminary results. J Radiat Res. 2011;52(6):812-20.

5. Tachimori Y. Role of salvage esophagectomy after definitive chemoradiotherapy. Gen Thorac Cardiovasc Surg. 2009;57(2):71-8. 
6. Shioyama Y, Nakamura K, Ohga S, et al. Radiation therapy for recurrent esophageal cancer after surgery: clinical results and prognostic factors. Jpn J Clin Oncol. 2007;37(12):918-23.

7. Yano M, Takachi K, Doki Y, et al. Prognosis of patients who develop cervical lymph node recurrence following curative resection for thoracic esophageal cancer. Dis Esophagus. 2006;19(2):73-7.

8. Jereczek-Fossa BA, Kowalczyk A, D'Onofrio A, et al. Three-dimensional conformal or stereotactic reirradiation of recurrent, metastatic or new primary tumors. Analysis of 108 patients. Strahlenther Onkol. 2008;184(1):36-40.

9. Lin SH, Chang JY. Esophageal cancer: diagnosis and management. Chin J Cancer. 2010;29(10):843-54.

10. Gabrail NY, Harrison BR, Sunwoo YC. Chemo-irradiation induced aortoesophageal fistula. J Surg Oncol. 1991;48(3):213-5.

11. Sivaraman SK, Drummond R. Radiation-induced aortoesophageal fistula: an unusual case of massive upper gastrointestinal bleeding. J Emerg Med. 2002;23(2):175-8.

12. Gudovsky LM, Koroleva NS, Biryukov YB, Chernousov AF, Perelman MI. Tracheoesophageal fistulas. Ann Thorac Surg. 1993;55(4):868-75.

13. Ohtsu A, Boku N, Muro K, et al. Definitive chemoradiotherapy for T4 and/ or M1 lymph node squamous cell carcinoma of the esophagus. J Clin Oncol. 1999;17(9):2915-21.

14. Zhang Y, Li Z, Zhang W, Chen W, Song Y. Risk factors for esophageal fistula in patients with locally advanced esophageal carcinoma receiving chemoradiotherapy. Onco Targets Ther. 2018;11:2311-7.

15. Huang W, Huang Y, Sun J, et al. Atlas of the thoracic lymph nodal delineation and recommendations for lymph nodal CTV of esophageal squamous cell cancer in radiation therapy from China. Radiother Oncol. 2015;116(1):100-6.

16. Hu B, Jia F, Zhou H, et al. Risk factors associated with esophageal fistula after radiotherapy for esophageal squamous cell carcinoma. J Cancer. 2020;1 1(12):3693-700

17. Sugahara S, Ohara K, Okumura T, Irie T, Nakajima K, Itai Y. Nihon Igaku Hoshasen Gakkai Zasshi. 1999;59(13):754-9.

18. Katano A, Yamashita H, Nakagawa K. Re-irradiation of locoregional esophageal cancer recurrence following definitive chemoradiotherapy: a report of 6 cases. Mol Clin Oncol. 2017;7(4):681-6.

19. Zhou ZG, Zhen CJ, Bai WW, et al. Salvage radiotherapy in patients with local recurrent esophageal cancer after radical radiochemotherapy. Radiat Oncol. 2015;10:54.

20. Xu Y, Wang L, He B, et al. Development and validation of a risk prediction model for radiotherapy-related esophageal fistula in esophageal cancer. Radiat Oncol. 2019;14(1):181.

21. Han X, Zhao YS, Fang Y, et al. Placement of transnasal drainage catheter and covered esophageal stent for the treatment of perforated esophageal carcinoma with mediastinal abscess. I Surg Oncol. 2016;114(6):725-30.

22. Nishimura Y, Suzuki M, Nakamatsu K, Kanamori S, Yagyu Y, Shigeoka $H$. Prospective trial of concurrent chemoradiotherapy with protracted infusion of 5-fluorouracil and cisplatin for T4 esophageal cancer with or without fistula. Int J Radiat Oncol Biol Phys. 2002;53(1):134-9.

23. Ishida K, Ando N, Yamamoto S, Ide H, Shinoda M. Phase II study of cisplatin and 5 -fluorouracil with concurrent radiotherapy in advanced squamous cell carcinoma of the esophagus: a Japan esophageal oncology group (JEOG)/Japan clinical oncology group trial (JCOG9516) [published correction appears in Jpn J Clin Oncol. 2005 Feb;35(2):108]. Jpn J Clin Oncol. 2004;34(10):615-9.

24. Zhang J, Peng F, Li N, et al. Salvage concurrent radio-chemotherapy for post-operative local recurrence of squamous-cell esophageal cancer. Radiat Oncol. 2012;7:93

25. Tsushima T, Mizusawa J, Sudo K, et al. Risk factors for esophageal fistula associated with chemoradiotherapy for locally advanced unresectable esophageal cancer: a supplementary analysis of JCOG0303. Medicine (Baltimore). 2016;95(20):e3699.

\section{Publisher's Note}

Springer Nature remains neutral with regard to jurisdictional claims in published maps and institutional affiliations.

Ready to submit your research? Choose BMC and benefit from:

- fast, convenient online submission

- thorough peer review by experienced researchers in your field

- rapid publication on acceptance

- support for research data, including large and complex data types

- gold Open Access which fosters wider collaboration and increased citations

- maximum visibility for your research: over 100M website views per year

At BMC, research is always in progress.

Learn more biomedcentral.com/submissions 